\title{
On a Problem of an Infinite Plate with a Curvilinear Hole inside the Unit Circle
}

\author{
F. S. Bayones, B. M. Alharbi \\ Department of Mathematics, Faculty of Science, Taif University, Taif, KSA \\ Email: F.S.Bayones@hotmail.com, shobee33@gmail.com
}

Received 8 January 2015; accepted 26 January 2015; published 29 January 2015

Copyright (C) 2015 by authors and Scientific Research Publishing Inc.

This work is licensed under the Creative Commons Attribution International License (CC BY). http://creativecommons.org/licenses/by/4.0/

c) (i) Open Access

\begin{abstract}
In this work, we used the complex variable methods to derive the Goursat functions for the first and second fundamental problem of an infinite plate with a curvilinear hole $C$. The hole is mapped in the domain inside a unit circle by means of the rational mapping function. Many special cases are discussed and established of these functions. Also, many applications and examples are considered. The results indicate that the infinite plate with a curvilinear hole inside the unit circle is very pronounced.
\end{abstract}

\section{Keywords}

Complex Variable Method, An Infinite Plate, Curvilinear Hole, Conformal Mapping, Goursat Functions

\section{Introduction}

Many intangible phenomena can be found in nature-like magnetic field, electricity and heat. These phenomena cannot be presented mathematically in the real plane. The complex plane plays an important role in presenting these intangible phenomena. Also, many mathematical problems cannot be solved in the real plane; their solutions can be found in the complex plane.

The considerable mathematical difficulties which arise during any attempt to solve plane elastic problems necessitate the search for practical methods of solution. The first use and development of the methods of complex function theory in two-dimensional elastic problems were made by Muskhelishvili (see [1]), and their ideas were expounded in their latter books (see [2]-[4]). The development of the theory was based on the complex representation of the general solution of the equations of the plane theory of elasticity. This complex representation has been found very useful for the effective solution of the plane elastic problems.

Contact and mixed problems in the theory of elasticity have been recognized as a rich and challenging subject 
for study (see Popov [5], Sabbah [6] and Atkin and Fox [7]). These problems can be established from the initial value problems or from the boundary value problems, or from the mixed problems (see Colton and Kress [8] and Abdou [9]). Also, many different methods are established for solving the contact and mixed problems in elastic and thermoelastic problems; the books edited by Noda [10], Hetnarski [11], Parkus [12] and Popov [5] contain many different methods to solve the problems in the theory of elasticity in one, two and three dimensions.

Several authors wrote about the boundary value problems and their applications in many different sciences (see [7] [13]-[15]). Form these problems, we established contact and mixed problems (see [8] [16]). Complex variable method used to express the solutions of these problems in the form of power series applied Laurent's theorem (see [8] [17]-[19]). The extensive literature on the topic is now available and we can only mention a few recent interesting investigations in [20]-[24].

The first and second fundamental problems in the plane theory of elasticity are equivalent to finding analytic functions $\phi_{1}(z)$ and $\psi_{1}(z)$ of one complex argument $z=x+i y$.

These functions satisfy the boundary conditions

$$
k \phi_{1}(t)-t \overline{\phi_{1}}(t)-\overline{\psi_{1}}(t)=f(t)
$$

where $\phi_{1}(t)$ and $\psi_{1}(t)$ are two analytic functions; $t$ denotes the affix of a point on the boundary. In the first fundamental problem $k=-1, f(t)$ is a given function of stresses, while in the second fundamental problem

$$
\begin{aligned}
& k=\chi=\frac{(\lambda+3 \mu)}{\lambda+\mu} \\
& \lambda=\frac{E}{(1-2 v)(1+v)}
\end{aligned}
$$

And $f=2 \mu \mathrm{g}$ is a given function of the displacement; $\lambda$ and $\mu$ are called the Lame constants.

Let the complex potentials $\phi_{1}(t)$ and $\psi_{1}(t)$ take the form

$$
\begin{aligned}
& \phi_{1}(\zeta)=-\frac{X+i Y}{2 \pi(1+\chi)} \ln \zeta+c \Gamma \zeta+\phi(\zeta) \\
& \psi_{1}(\zeta)=\chi \frac{(X-i Y)}{2 \pi(1+\chi)} \ln \zeta+c \Gamma^{*} \zeta+\psi(\zeta)
\end{aligned}
$$

where $X, Y$ are the components of the resultant vector of all external forces acting on the boundary and $\Gamma, \Gamma^{*}$ are constants; generally complex functions $\phi(\zeta), \psi(\zeta)$ are single-valued analytic functions within the region inside the unit circle $\gamma$ and $\phi(\infty)=0$.

Take the conformal mapping which mapped the domain of the curvilinear hole $C$ on the domain inside a unit circle $\gamma$ by the rational function

$$
z=w(\zeta),|\zeta|<1, c>0
$$

and $w^{\prime}(\zeta)$ does not vanish or become infinite to conform the curvilinear hole of an infinite elastic plate onto the domain inside a unit circle $\gamma$ i.e.

$$
w^{\prime}(\zeta) \neq 0, \infty
$$

\section{Conformal Mapping}

Consider the rational mapping on the domain inside a unit circle $\gamma$ by the rational function

$$
z=w(\zeta)=\frac{\zeta^{3}+m \zeta}{\zeta-n},|n|<1,|\zeta|<1,
$$

where, $m$ and $n$ are complex number $n=n_{1}+i n_{2}, m=m_{1}+i m_{2}$, Equation (7) must satisfy the condition Equation (6). 
For determining the tax parameters $x$ and $y$, we put $\zeta=\rho \mathrm{e}^{\mathrm{i} \theta},|\rho|=1$ in Equation (7) to get

$$
x+i y=\frac{\left(\cos 3 \theta+m_{1} \cos \theta-m_{2} \sin \theta\right)+i\left(\sin 3 \theta+m_{1} \sin \theta+m_{2} \cos \theta\right)}{\left(\cos \theta-n_{1}\right)+i\left(\sin \theta-n_{2}\right)}
$$

Then

$$
\begin{aligned}
& x=\frac{\cos 2 \theta+m_{1}-n_{1}\left(\cos 3 \theta+m_{1} \cos \theta-m_{2} \sin \theta\right)+n_{1}\left(\sin 3 \theta+m_{1} \sin \theta+m_{2} \cos \theta\right)}{\left(\cos \theta-n_{1}\right)^{2}+\left(\sin \theta-n_{2}\right)^{2}} \\
& y=\frac{\sin 2 \theta+m_{2}+n_{2}\left(\cos 3 \theta+m_{1} \cos \theta-m_{2} \sin \theta\right)-n_{1}\left(\sin 3 \theta+m_{1} \sin \theta+m_{2} \cos \theta\right)}{\left(\cos \theta-n_{1}\right)^{2}+\left(\sin \theta-n_{2}\right)^{2}}
\end{aligned}
$$

Also,

$$
z^{\prime}=w^{\prime}(\zeta)=\frac{2 \zeta^{3}-3 n \zeta^{2}-m n}{(\zeta-n)^{2}}
$$

To obtain the critical points, we consider

$$
2 \zeta^{3}-3 n \zeta^{2}-m n=0
$$

this linear equation of three order, the roots of this equation must be under 1 .

The following graphs give the different shapes of the rational mapping (7), see Figure 1.

\section{The Components of Stresses}

It is known that, the components of stresses are given by, see [1]

$$
\begin{aligned}
& \sigma_{x x}+\sigma_{y y}=4 \operatorname{Re}\left\{\phi^{\prime}(z)\right\} \\
& \sigma_{y y}-\sigma_{x x}+i \sigma_{x y}=2\left\{\bar{z} \phi^{\prime \prime}(z)+\psi^{\prime}(z)\right\}
\end{aligned}
$$

Hence, we have

$$
\begin{gathered}
\sigma_{y y}=\operatorname{Re}\left\{2 \phi^{\prime}(z)+M(z, \bar{z})\right\}, M(z, \bar{z})=\bar{z} \phi^{\prime \prime}(z)+\psi^{\prime}(z) \\
\sigma_{x x}=\operatorname{Re}\left\{2 \phi^{\prime}(z)-M(z, \bar{z})\right\}, M(z, \bar{z})=\bar{z} \phi^{\prime \prime}(z)+\psi^{\prime}(z)
\end{gathered}
$$

and

$$
\sigma_{x y}=2 \operatorname{Im}\left\{\bar{z} \phi^{\prime \prime}(z)+\psi^{\prime}(z)\right\}=2 \operatorname{Im}\{M(z, \bar{z})\}
$$

\section{Goursat Functions}

To obtain the tow complex potential functions (Goursat functions) by using the conformal mapping (7) in the boundary condition (6). We write the expression $\frac{w(\zeta)}{\overline{w^{\prime}(\zeta)}}$ in the form,

$$
\frac{w(\zeta)}{\overline{w^{\prime}(\zeta)}}=\alpha(\zeta)+\overline{\beta(\zeta)}
$$

where,

$$
\alpha(\zeta)=\frac{h}{(\zeta-n)}, \overline{\beta(\zeta)}=\frac{w(\zeta)}{\overline{w^{\prime}(\zeta)}}-\frac{h}{\zeta-n}
$$



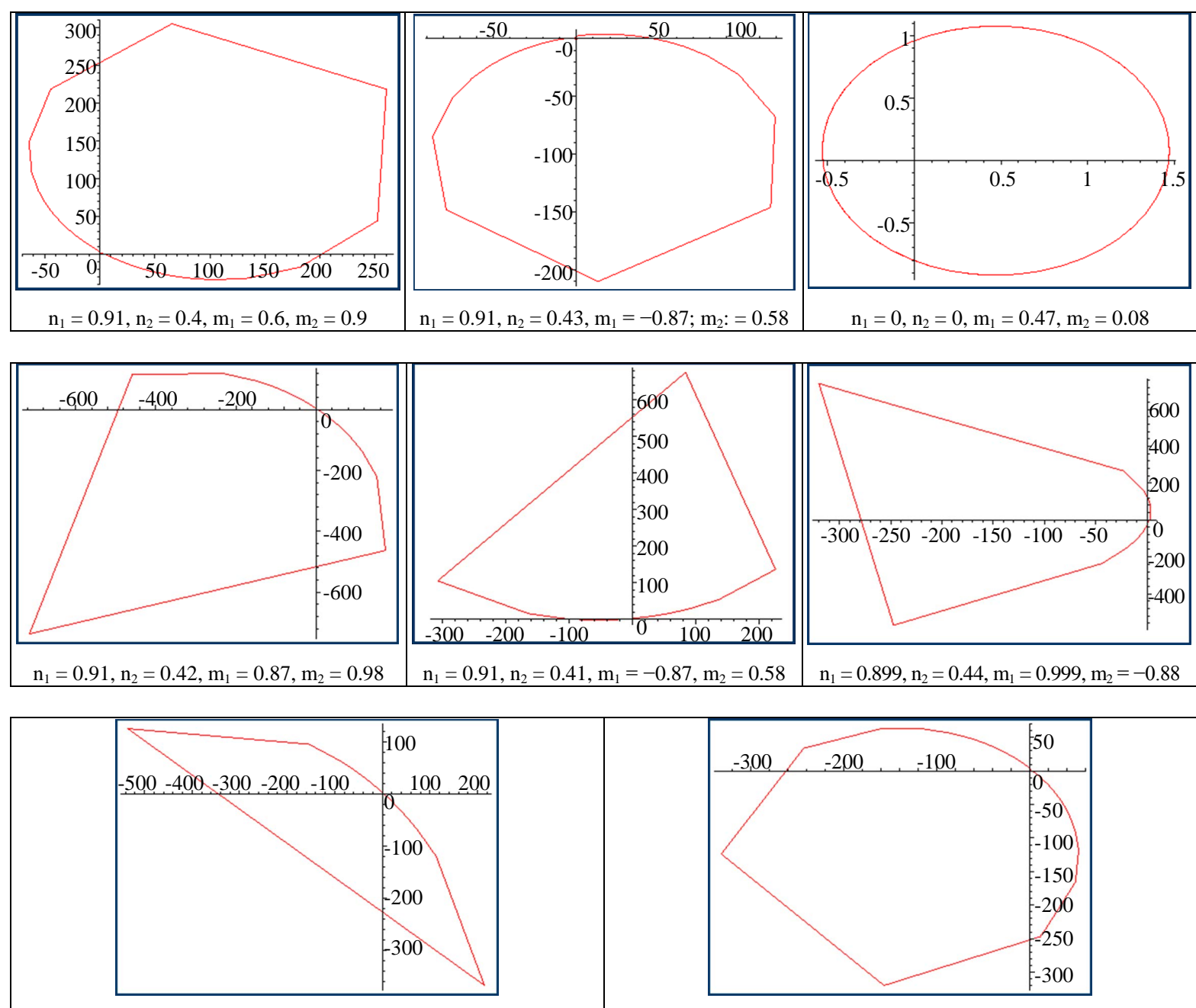

$\mathrm{n}_{1}=0.899, \mathrm{n}_{2}=0.44, \mathrm{~m}_{1}=0.09, \mathrm{~m}_{2}=0.058$

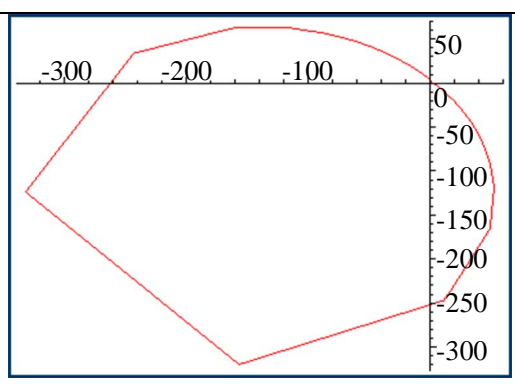

$\mathrm{n}_{1}=-0.899, \mathrm{n}_{2}=-0.45, \mathrm{~m}_{1}=0.9, \mathrm{~m}_{2}=0.58$

Figure 1. The different shapes of the rational mapping (7).

$\beta\left(\zeta^{-1}\right)$ is a regular function for $|\zeta|<1$.

In order to separate the singularity, we use the definition of mapping, to have

$$
\frac{w(\zeta)}{\overline{w^{\prime}(\zeta)}}=\frac{\zeta^{3}+m \zeta}{\zeta-n} \cdot \frac{\zeta(1-n \zeta)^{2}}{\left(2-3 n \zeta-m n \zeta^{3}\right)}=\frac{1}{\zeta-n} \cdot \frac{\zeta^{2}\left(\zeta^{2}+m\right)(1-n \zeta)^{2}}{\left(2-3 n \zeta-m n \zeta^{3}\right)}
$$

The term $\left(2-3 n \zeta-m n \zeta^{3}\right)$ in the are has no singular point while $(\zeta-n)$ has a singularity at $\zeta=n$. where

$$
\overline{w^{\prime}(\zeta)}=\frac{\left(2-3 n \zeta-m n \zeta^{3}\right)}{\zeta(1-n \zeta)^{2}}
$$

To determine $h$ form Equation (19), we can write the form

$$
\frac{w(\sigma)}{\overline{w^{\prime}(\sigma)}}=\frac{1}{\sigma-n} \cdot \frac{\sigma^{2}\left(\sigma^{2}+m\right)(1-n \sigma)^{2}}{\left(2-3 n \sigma-m n \sigma^{3}\right)} .
$$

By using the residues in this equating we have 


$$
h=\frac{n^{2}\left(n^{2}+m\right)\left(1-n^{2}\right)^{2}}{\left(2-3 n^{2}-m n^{4}\right)} .
$$

Using Equation (3) and Equation (4) in Equation (1), we get

$$
k \phi(\sigma)-\alpha(\sigma) \overline{\phi^{\prime}(\sigma)}-\overline{\psi_{*}(\sigma)}=G(\sigma)
$$

where

$$
\begin{aligned}
& \psi_{*}(\sigma)=\psi(\sigma)+\beta(\sigma) \phi^{\prime}(\sigma) \\
& G(\sigma)=F(\sigma)-c k \Gamma \sigma+\frac{c \overline{\Gamma^{*}}}{\sigma}+N(\sigma) \alpha(\sigma)+N(\sigma) \overline{\beta(\sigma)} \\
& N(\sigma)=\left[c \bar{\Gamma}-\frac{\sigma(X-i Y)}{2 \pi(1+\chi)}\right], \quad F(\sigma)=f(t)
\end{aligned}
$$

Assume that the function $F(\sigma)$ with its derivatives must satisfy the Holder condition. Our aim is to determine the functions $\phi(\zeta)$ and $\psi(\zeta)$ for the various boundary value problems. For this multiply both sides of Equation (23) by $\frac{\mathrm{d} \sigma}{2 \pi i(\sigma-\zeta)}$, where $\zeta$ is any point in the interior of $\gamma$ and integral over the circle, we obtain

$$
\frac{k}{2 \pi i} \int_{\gamma} \frac{\phi(\sigma)}{\sigma-\zeta} \mathrm{d} \sigma-\frac{1}{2 \pi i} \int_{\gamma} \frac{\alpha(\sigma) \overline{\phi^{\prime}}(\sigma)}{\sigma-\zeta} \mathrm{d} \sigma-\frac{1}{2 \pi i} \int_{\gamma} \frac{\overline{\psi_{*}}(\sigma)}{\sigma-\zeta} \mathrm{d} \sigma=\frac{1}{2 \pi i} \int_{\gamma} \frac{G(\sigma)}{\sigma-\zeta} \mathrm{d} \sigma
$$

Using Equations (24)-(26) in Equation (27) then applying the properties of Cauchy integral, to have

$$
\frac{k}{2 \pi i} \int_{\gamma} \frac{\phi(\sigma)}{\sigma-\zeta} \mathrm{d} \sigma=-k \phi(\zeta)
$$

and

$$
\begin{aligned}
& \frac{1}{2 \pi i} \int_{\gamma} \frac{\alpha(\sigma) \overline{\phi^{\prime}}(\sigma)}{\sigma-\zeta} \mathrm{d} \sigma=\frac{c h b}{n-\zeta} \\
& \frac{1}{2 \pi i} \int_{\gamma} \frac{N(\sigma) \alpha(\sigma)}{(\sigma-\zeta)} \mathrm{d} \sigma=\frac{N(n) h}{n-\zeta}
\end{aligned}
$$

Also,

$$
\frac{1}{2 \pi i} \int_{\gamma} \frac{G(\sigma)}{(\sigma-\zeta)} \mathrm{d} \sigma=A(\zeta)-\frac{c \overline{\Gamma^{*}}}{\zeta}+\frac{h N(n)}{(n-\zeta)}
$$

where,

$$
A(\zeta)=\frac{1}{2 \pi i} \int_{\gamma} \frac{F(\sigma)}{(\sigma-\zeta)} \mathrm{d} \sigma, N(\sigma)=\left[c \bar{\Gamma}-\frac{\sigma(X-i Y)}{2 \pi(1+\chi)}\right] .
$$

From the above, Equation (27) becomes

$$
-k \phi(\zeta)=A(\zeta)+\frac{h}{n-\zeta}(c b+N(n))-\frac{c \overline{\Gamma^{*}}}{\zeta}
$$


To determined $b$, where $b$ are complex constants, differentiating Equation (33) with respect to $\zeta$ and substituting in Equation (29), we get

$$
\frac{1}{2 \pi i} \int_{\gamma} \frac{\alpha(\sigma)}{(\sigma-\zeta)}\left[-\overline{A^{\prime}(\sigma)}-c \Gamma^{*} \sigma^{2}-\frac{h \sigma^{2}}{(n \sigma-1)^{2}}(c \bar{b}+\overline{N(n)}) \mathrm{d} \sigma\right]=\frac{c k h b}{(n-\zeta)}
$$

Substituting Equation (18) in Equation (34), then using the properties of Cauchy integral and applying the reside theorem at the singular points, we obtain

$$
c k b+\overline{A^{\prime}(n)}+c \Gamma^{*} n^{2}+v h(c \bar{b}+\overline{N(n)})=0
$$

where

$$
v=\frac{n^{2}}{\left(1-n^{2}\right)^{2}}
$$

The last equation can be written in the form

$$
c k b+v h c \bar{b}=E
$$

where,

$$
E=-\overline{A^{\prime}(n)}-c \Gamma^{*} n^{2}-v h \overline{N(n)}
$$

taking the complex conjugate of Equation (37), we get

$$
c k \bar{b}+v h c b=\bar{E}
$$

form Equation (37) and Equation (39), we have

$$
b=\frac{k E-v h \bar{E}}{c\left(k^{2}-v^{2} h^{2}\right)}
$$

To obtain the complex function $\psi(\zeta)$ we have form Equation (23) after substituting the expression of $\psi(\sigma)$ and $G(\sigma)$, and taking the complex conjugate of the resulting equation after using the expression of $\overline{\beta(\sigma)}$ to yields,

$$
\psi(\sigma)=-\overline{F(\sigma)}+c k \bar{\Gamma} \sigma^{-1}-c \Gamma^{*} \sigma+k \overline{\phi(\sigma)}-\overline{\alpha(\sigma)} \phi_{*}(\sigma)-\frac{\overline{w(\sigma)}}{w^{\prime}(\sigma)} \phi_{*}(\sigma)+\frac{h \sigma}{(1-n \sigma)} \phi_{*}(\sigma)
$$

where,

$$
\phi_{*}(\sigma)=\phi^{\prime}(\sigma)+\overline{N(\sigma)}, \overline{N(\sigma)}=\left[c \Gamma-\frac{\sigma^{-1}(X+i Y)}{2 \pi(1+\chi)}\right]
$$

and calculate sum residue, we obtain multiplying both sides of Equation (41) by $\frac{1}{2 \pi i(\sigma-\zeta)}$, where $\zeta$ is any point in the interior of $\gamma$ and integrating over the circle, then using the properties of Cauchy's integral and calculating the sum residue, we obtain

$$
\psi(\zeta)=c k \bar{\Gamma} \zeta^{-1}-\frac{\overline{w(\zeta)}}{w^{\prime}(\zeta)} \phi_{*}(\zeta)+\frac{h \zeta}{(1-n \zeta)} \phi_{*}\left(n^{-1}\right)+B(\zeta)-B
$$

where, 


$$
B(\zeta)=\frac{1}{2 \pi i} \int \frac{\overline{F(\sigma)}}{(\sigma-\zeta)} \mathrm{d} \sigma,
$$

and

$$
B=\frac{1}{2 \pi i} \int_{\gamma} \frac{\overline{F(\sigma)}}{\sigma} \mathrm{d} \sigma .
$$

\section{Special Cases}

Now, we are in a position to consider several cases:

1) Let $m=0, n \neq 0$, we get the mapping function represent of the hole is an ellipse, see Figure 2

$$
z=w(\zeta)=\frac{\zeta^{3}}{\zeta-n}
$$

by let

$$
z^{\prime}=0 \Rightarrow 2 \zeta^{3}-3 n \zeta^{2}=0
$$
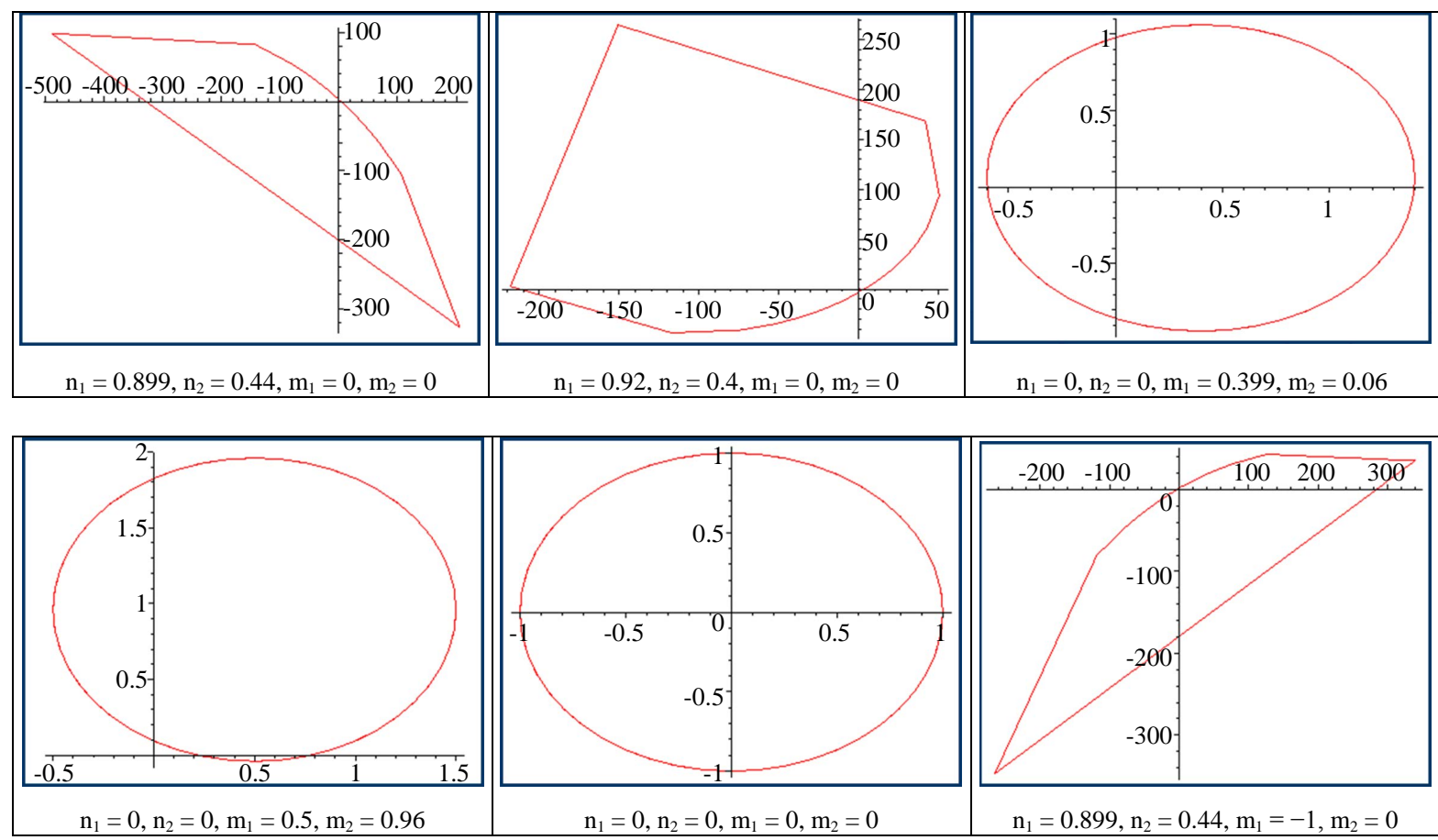

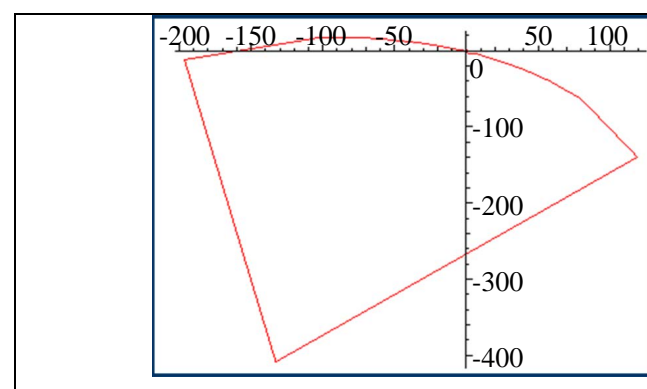

$\mathrm{n}_{1}=0.91, \mathrm{n}_{2}=-0.41, \mathrm{~m}_{1}=-1, \mathrm{~m}_{2}=0$

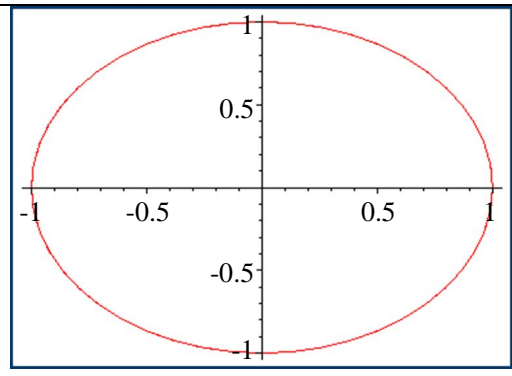

$\mathrm{n}_{1}=0.1 \times 10^{-10}, \mathrm{n}_{2}=0.5 \times 10^{-10}$

Figure 2. The different shapes of the rational mapping for special cases. 
Then (33) and (43) becomes

$$
\begin{aligned}
& h=\frac{n^{4}\left(1-n^{2}\right)^{2}}{2-3 n^{2}} \\
& -k \phi(\zeta)=A(\zeta)-\frac{c \overline{\Gamma^{*}}}{\zeta}+\frac{n^{4}\left(1-n^{2}\right)^{2}}{\left(2-3 n^{2}\right)(n-\zeta)}\left[N(n)+\frac{k E-h v \bar{E}}{k^{2}-h^{2} v^{2}}\right] \\
& -k \phi(\zeta)=A(\zeta)-\frac{c \overline{\Gamma^{*}}}{\zeta}+\frac{n^{4}\left(1-n^{2}\right)^{2}}{\left(2-3 n^{2}\right)(n-\zeta)}\left[N(n)+\frac{k E-\frac{n^{6} \bar{E}}{\left(2-3 n^{2}\right)}}{\left.k^{2}-\frac{n^{12}}{\left(2-3 n^{2}\right)^{2}}\right]}\right.
\end{aligned}
$$

Also,

$$
\psi(\zeta)=B(\zeta)+\frac{c k \bar{\Gamma}}{\zeta}-\frac{w\left(\zeta^{-1}\right)}{w^{\prime}(\zeta)} \phi_{*}(\zeta)+\frac{n^{4}\left(1-n^{2}\right)^{2} \zeta}{\left(2-3 n^{2}\right)(1-n \zeta)} \phi_{*}\left(n^{-1}\right)-B
$$

where

$$
E=-\overline{A^{\prime}(n)}-c \Gamma^{*} n^{2}-\frac{n^{6}}{2-3 n^{2}} \overline{N(n)}
$$

2) For $n=0,0 \leq m \leq 1$, we get the mapping function represent of the hole is an ellipse, see Figure 2

$$
\begin{aligned}
& z=\frac{\zeta^{3}+m \zeta}{\zeta}=\zeta^{2}+m \\
& z^{\prime}=0 \Rightarrow 2 \zeta=0
\end{aligned}
$$

then

$$
\zeta=\mathrm{e}^{i \alpha}, \mathrm{e}^{i \alpha}=\cos \alpha+i \sin \alpha
$$

Then (33) and (43) becomes

$$
\begin{gathered}
h=0 \\
-k \phi(\zeta)=A(\zeta)-\frac{c \overline{\Gamma^{*}}}{\zeta} \\
\psi(\zeta)=B(\zeta)+\frac{c k \bar{\Gamma}}{\zeta}-\frac{1+m \zeta^{2}}{2 \zeta^{3}} \phi_{*}(\zeta)-B
\end{gathered}
$$

where

$$
E=-\overline{A^{\prime}(n)}-c \Gamma^{*} n^{2}, n=0 .
$$

3) Let $m=n=0$, we get the mapping function represent of the hole is an ellipse, see Figure 2

$$
\begin{gathered}
z=\frac{\zeta^{3}}{\zeta}=\zeta^{2} \\
z^{\prime}=0 \Rightarrow 2 \zeta=0 \\
\zeta=\mathrm{e}^{i \alpha}, \mathrm{e}^{i \alpha}=\cos \alpha+i \sin \alpha
\end{gathered}
$$


Then (33) and (43) becomes

$$
\begin{gathered}
h=0 \\
-k \phi(\zeta)=A(\zeta)-\frac{c \overline{\Gamma^{*}}}{\zeta} \\
\psi(\zeta)=B(\zeta)+\frac{c k \bar{\Gamma}}{\zeta}-\frac{1}{2 \zeta^{3}} \phi_{*}(\zeta)-B \\
E=-\overline{A^{\prime}(n)}-c \Gamma^{*} n^{2}, n=0 .
\end{gathered}
$$

4) Let $m=-1$, where $m_{1}=-1, m_{2}=0$ we get the mapping function represent of the hole is an ellipse, see Figure 2

$$
\begin{gathered}
z=\frac{\zeta^{3}-\zeta}{\zeta-n} \\
z^{\prime}=0 \Rightarrow 2 \zeta^{3}-3 n \zeta^{2}+n=0
\end{gathered}
$$

Then (33) and (43) becomes

$$
\begin{gathered}
h=\frac{n^{2}\left(n^{2}-1\right)\left(1-n^{2}\right)^{2}}{2-3 n^{2}+n^{4}} \\
-k \phi(\zeta)=A(\zeta)-\frac{c \overline{\Gamma^{*}}}{\zeta}+\frac{n^{2}\left(n^{2}-1\right)\left(1-n^{2}\right)^{2}}{\left(2-3 n^{2}+n^{4}\right)(n-\zeta)}\left[N(n)+\frac{k E-\frac{n^{4}\left(n^{2}-1\right) \bar{E}}{\left(2-3 n^{2}+n^{4}\right)}}{k^{2}-\frac{n^{8}\left(n^{2}-1\right)^{2}}{\left(2-3 n^{2}+n^{4}\right)^{2}}}\right]
\end{gathered}
$$

Also,

$$
\psi(\zeta)=B(\zeta)+\frac{c k \bar{\Gamma}}{\zeta}-\frac{w\left(\zeta^{-1}\right)}{w^{\prime}(\zeta)} \phi_{*}(\zeta)+\frac{n^{2}\left(n^{2}-1\right)\left(1-n^{2}\right)^{2} \zeta}{\left(2-3 n^{2}+n^{4}\right)(1-n \zeta)} \phi_{*}\left(n^{-1}\right)-B
$$

where

$$
E=-\overline{A^{\prime}(n)}-c \Gamma^{*} n^{2}+\frac{n^{4}\left(n^{2}-1\right)}{2-3 n^{2}+n^{4}} \overline{N(n)}
$$

5) Let $m=-n^{2}$,we get the mapping function represent of the hole is an ellipse, see Figure 2

$$
\begin{gathered}
z=\frac{\zeta^{3}-n^{2} \zeta}{\zeta-n}=\zeta^{2}+n \zeta \\
z^{\prime}=0 \Rightarrow 2 \zeta+n=0 \\
\zeta=-\frac{n}{2}
\end{gathered}
$$

Then (33) and (43) becomes

$$
h=0
$$




$$
-k \phi(\zeta)=A(\zeta)-\frac{c \overline{\Gamma^{*}}}{\zeta}
$$

Also,

$$
\begin{gathered}
\psi(\zeta)=B(\zeta)+\frac{c k \bar{\Gamma}}{\zeta}-\frac{w\left(\zeta^{-1}\right)}{w^{\prime}(\zeta)} \phi_{*}(\zeta)-B \\
E=-\overline{A^{\prime}(n)}-c \Gamma^{*} n^{2}+\frac{n^{4}\left(n^{2}+1\right)}{2-3 n^{2}+n^{4}} \overline{N(n)}
\end{gathered}
$$

\section{Applications}

In this section we study some applications:

1) For $k=-1, \Gamma=\frac{p}{4}, \Gamma^{*}=-\frac{1}{2} p \mathrm{e}^{-2 i \theta}$ and $X=Y=f=0$, we have the case of infinite plate stretched at infinity by the application of a uniform tensile stress of intensity $p$, making an angle $\theta$ with the x-axis. The plate weakened by the curvilinear hole $C$ which is free from stresses (see Figure 3, Figure $4\left(n_{1}=0.001, n_{2}=\right.$ $0.002 l, m_{1}=0.025, m_{2}=0.03 I, c=2, p=0.25$ )). Then the functions in (33) and (43) become

$$
\begin{gathered}
f=0 \Rightarrow A(\zeta)=0 \\
N(n)=\left[c \bar{\Gamma}-\frac{n(X-i Y)]}{2 \pi(1+\chi)}\right]=\frac{c p}{4} \\
E=\frac{2 c n^{2} p \mathrm{e}^{-2 i \theta}-v h c p}{4}, \bar{E}=\frac{2 c n^{2} p \mathrm{e}^{2 i \theta}-v h c p}{4} \\
c b=\frac{k E-v h \bar{E}}{\left(k^{2}-v^{2} h^{2}\right)}=\frac{-E-v h \bar{E}}{\left(1-v^{2} h^{2}\right)} \\
\phi(\zeta)=\frac{c p \mathrm{e}^{2 i \theta}}{2 \zeta}+\frac{c h p}{4(n-\zeta)}\left(1+\frac{c h v-2 c n^{2} \mathrm{e}^{-2 i \theta}-2 h v c n^{2} \mathrm{e}^{2 i \theta}+c h^{2} v^{2}}{1-h^{2} v^{2}}\right) .
\end{gathered}
$$

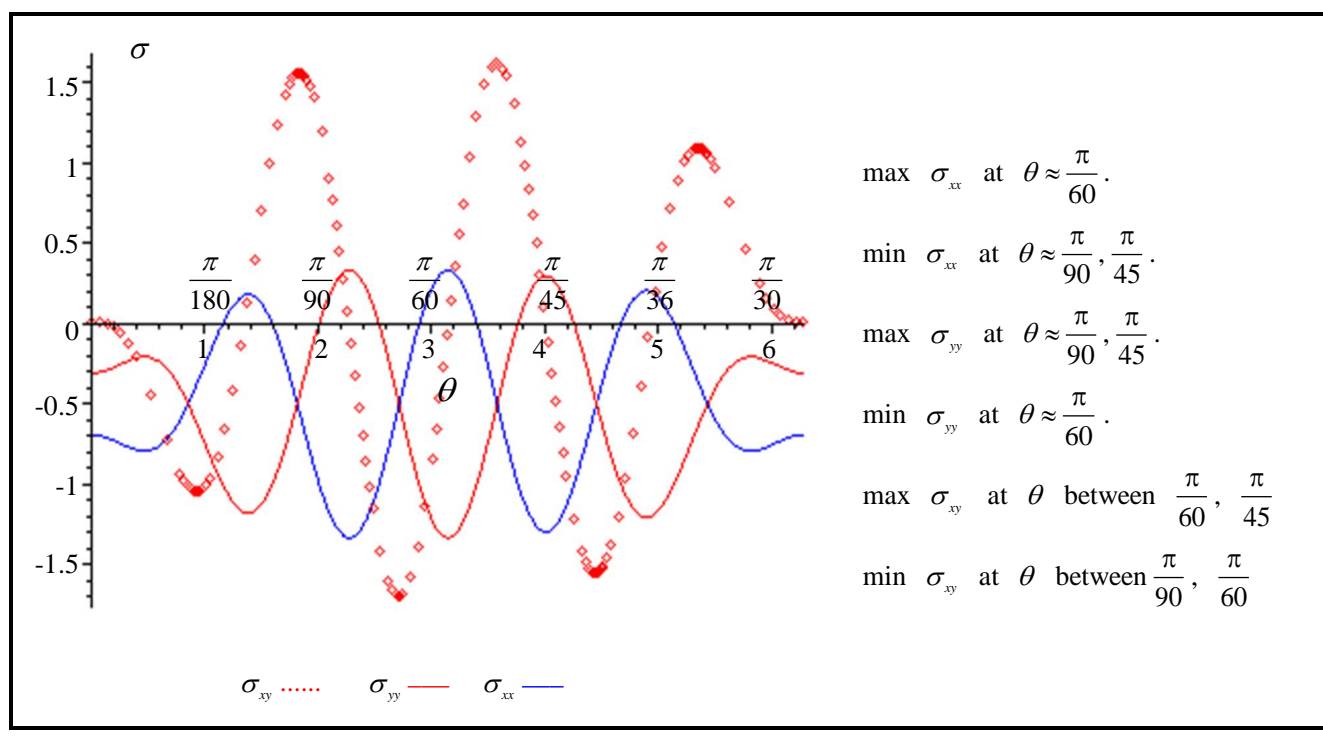

Figure 3. The relation between components of stresses and the angle made on the x-axis. 


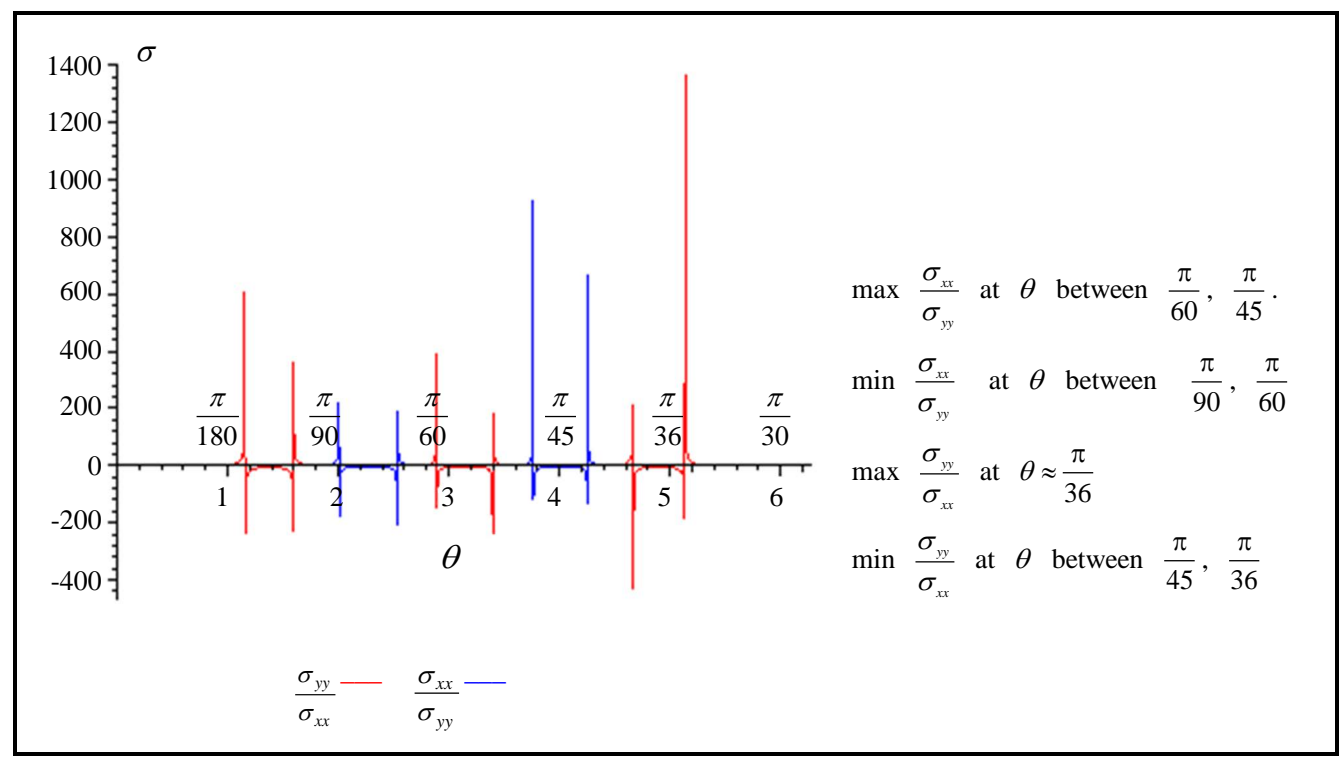

Figure 4. The ratio of vertical to horizontal stresses.

$$
\psi(\zeta)=-\frac{c p}{4 \zeta}-\frac{w\left(\zeta^{-1}\right)}{w^{\prime}(\zeta)} \phi_{*}(\zeta)+\frac{h \zeta}{(1-n \zeta)} \phi_{*}\left(n^{-1}\right)
$$

where

$$
\phi_{*}(\zeta)=\phi^{\prime}(\zeta)+\frac{c p}{4}
$$

2) For $k=-1, \Gamma=\Gamma^{*}=X=Y=0$ and $f=P t$, where $P$ is a real constant (see Figure 5, Figure 6 $\left(n_{1}=0.001, n_{2}=0.002 I, m_{1}=0.025, m_{2}=0.03 I, c=2, p=0.25\right)$ ).

Then the functions in (33) and (43) become

$$
\begin{aligned}
& f=P t \Rightarrow f=\frac{P c\left(\sigma^{3}+m \sigma\right)}{(\sigma-n)} \\
& \bar{f}=\frac{c P\left(1+m \sigma^{2}\right)}{\sigma^{2}(1-n \sigma)} \\
& A(\zeta)=\frac{c P}{2 \pi i} \int_{\gamma} \frac{\sigma^{3}+m \sigma}{(\sigma-n)(\sigma-\zeta)} \mathrm{d} \sigma=\frac{c P\left(n^{3}+m n\right)}{(n-\zeta)} \\
& A^{\prime}(\zeta)=\frac{c P\left(n^{3}+m n\right)}{(n-\zeta)^{2}}, \overline{A^{\prime}(\zeta)}=\frac{c P \zeta^{2}\left(n^{3}+m n\right)}{(n \zeta-1)^{2}} \\
& \overline{A^{\prime}(n)}=\frac{c P n^{2}\left(n^{3}+m n\right)}{\left(n^{2}-1\right)^{2}}, N(n)=0 \\
& E=-\frac{c P n^{2}\left(n^{3}+m n\right)}{\left(n^{2}-1\right)^{2}}=\bar{E}
\end{aligned}
$$




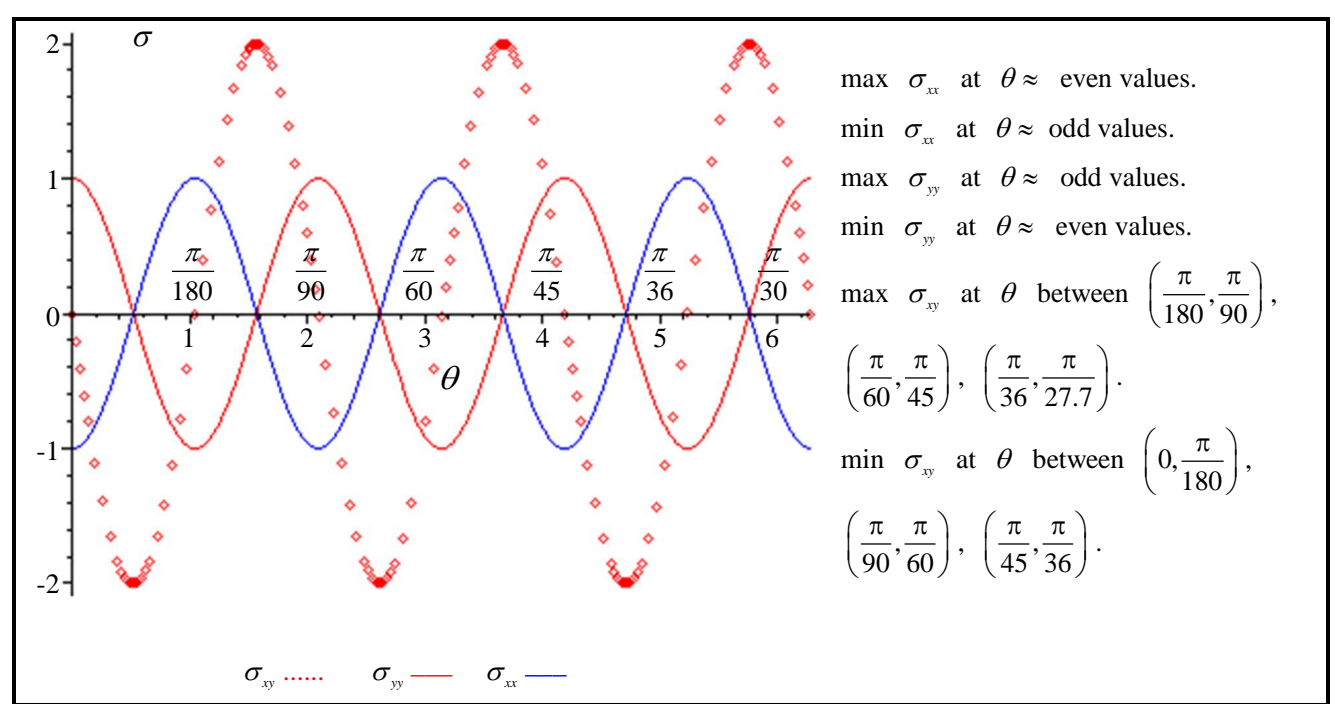

Figure 5. The relation between components of stresses and the angle made on the $\mathrm{x}$-axis.

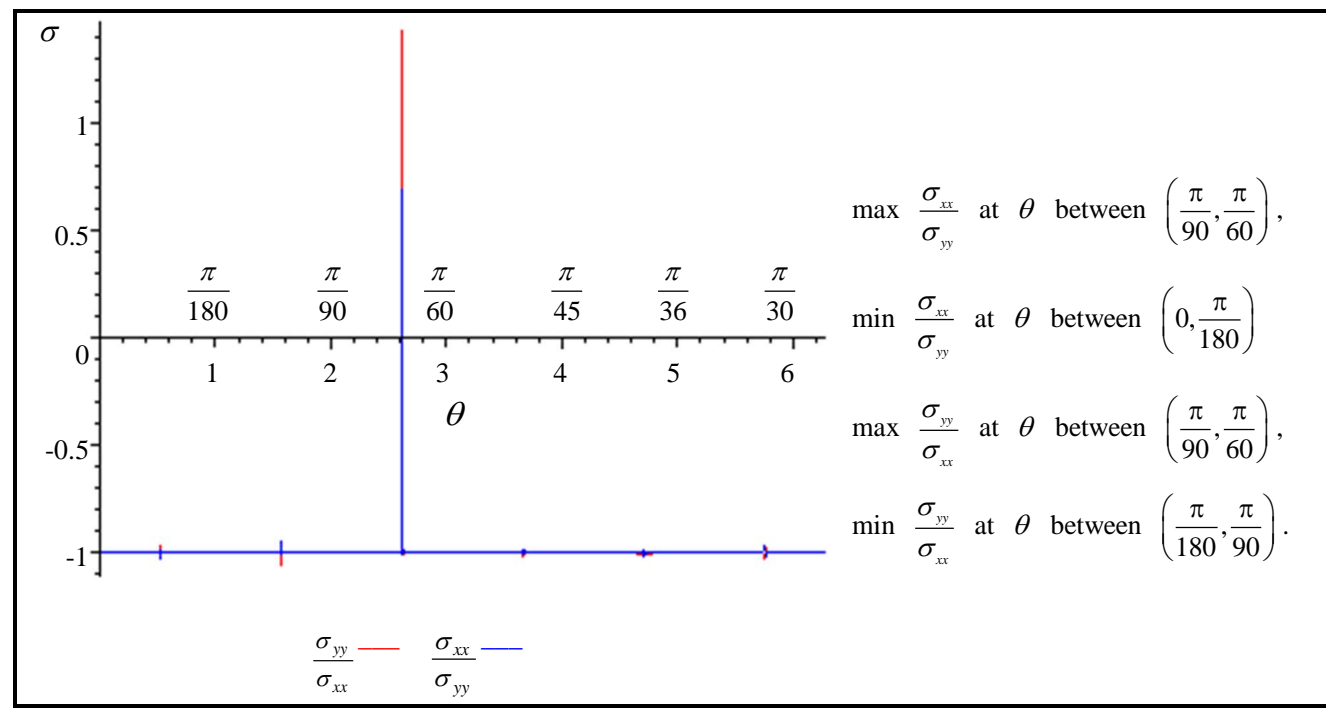

Figure 6. The ratio of vertical to horizontal stresses.

$$
\begin{aligned}
& c b=\frac{c P n^{2}\left(n^{3}+m n\right)}{(1-v h)\left(n^{2}-1\right)^{2}} \\
& \phi(\zeta)=\frac{c P\left(n^{3}+m n\right)}{(n-\zeta)}+\frac{h c P n^{2}\left(n^{3}+m n\right)}{(n-\zeta)(1-v h)\left(n^{2}-1\right)^{2}} \\
& \psi(\zeta)=-c P\left(n+\frac{1}{\zeta^{2}}\right)-\frac{w\left(\zeta^{-1}\right)}{w^{\prime}(\zeta)} \phi^{\prime}(\zeta)+\frac{h \zeta}{(1-n \zeta)} \phi^{\prime}\left(n^{-1}\right)
\end{aligned}
$$

where

$$
B(\zeta)=\frac{1}{2 \pi i} \int_{\gamma} \frac{\overline{F(\sigma)}}{(\sigma-\zeta)} \mathrm{d} \sigma=\frac{c P}{2 \pi i} \int_{\gamma} \frac{\left(1+m \sigma^{2}\right)}{\sigma^{2}(1-n \sigma)(\sigma-\zeta)} \mathrm{d} \sigma=-\frac{c P(1+n \zeta)}{\zeta^{2}}
$$




$$
B=\frac{c P}{2 \pi i} \int_{\gamma} \frac{\left(1+m \sigma^{2}\right)}{\sigma^{3}(1-n \sigma)} \mathrm{d} \sigma=2 c P\left(m+n^{2}\right), \phi_{*}(\zeta)=\phi^{\prime}(\zeta) .
$$

3) For $k=\chi, \Gamma=\Gamma^{*}=f=0$ (see Figure 7, Figure $8\left(n_{1}=0.001, n_{2}=0.002 I, m_{1}=0.025, m_{2}=0.03 I\right.$, $c=2, x=0.25, X=2, Y=2)$ ). Then the functions in (33) and (43) become

$$
\begin{gathered}
f=0 \Rightarrow A(\zeta)=0 \\
N(n)=-\frac{n(X-i Y)}{2 \pi(1+\chi)} \\
E=\frac{v h n(X+i Y)}{2 \pi(1+\chi)}, \bar{E}=\frac{v h n(X-i Y)}{2 \pi(1+\chi)}
\end{gathered}
$$

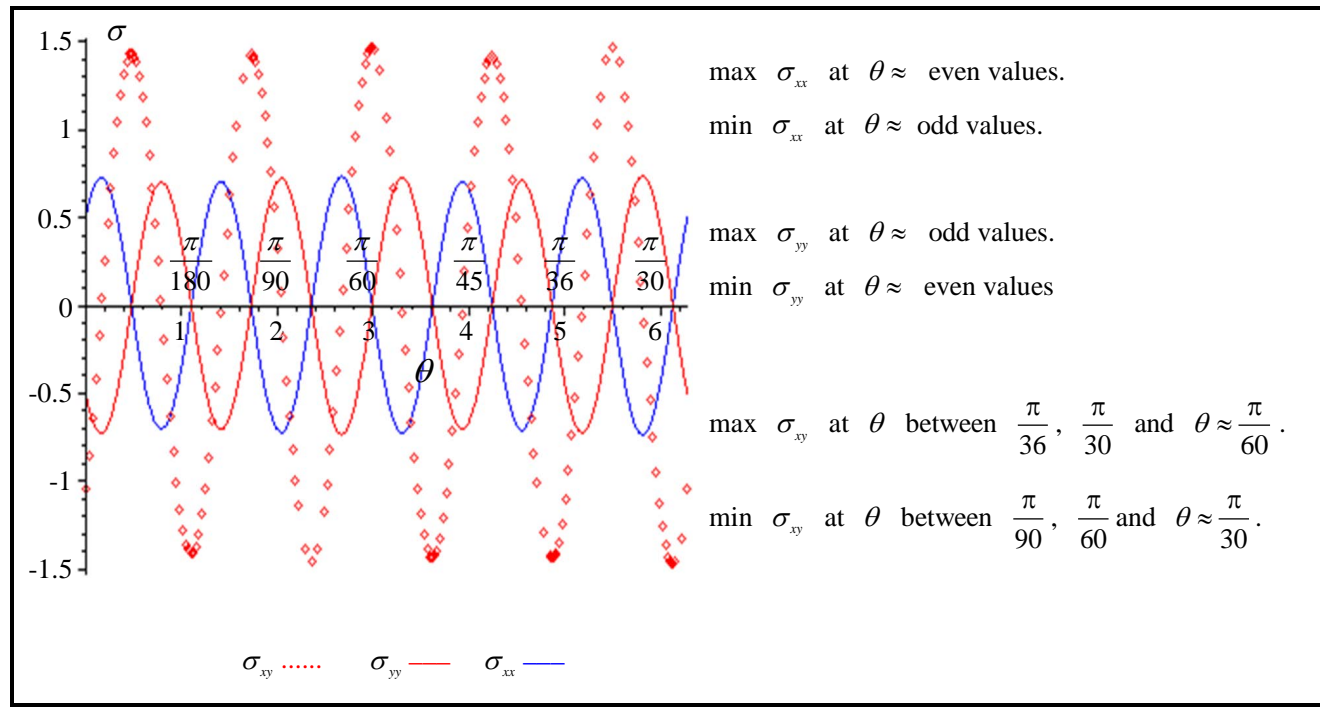

Figure 7. The relation between components of stresses and the angle made on the x-axis.

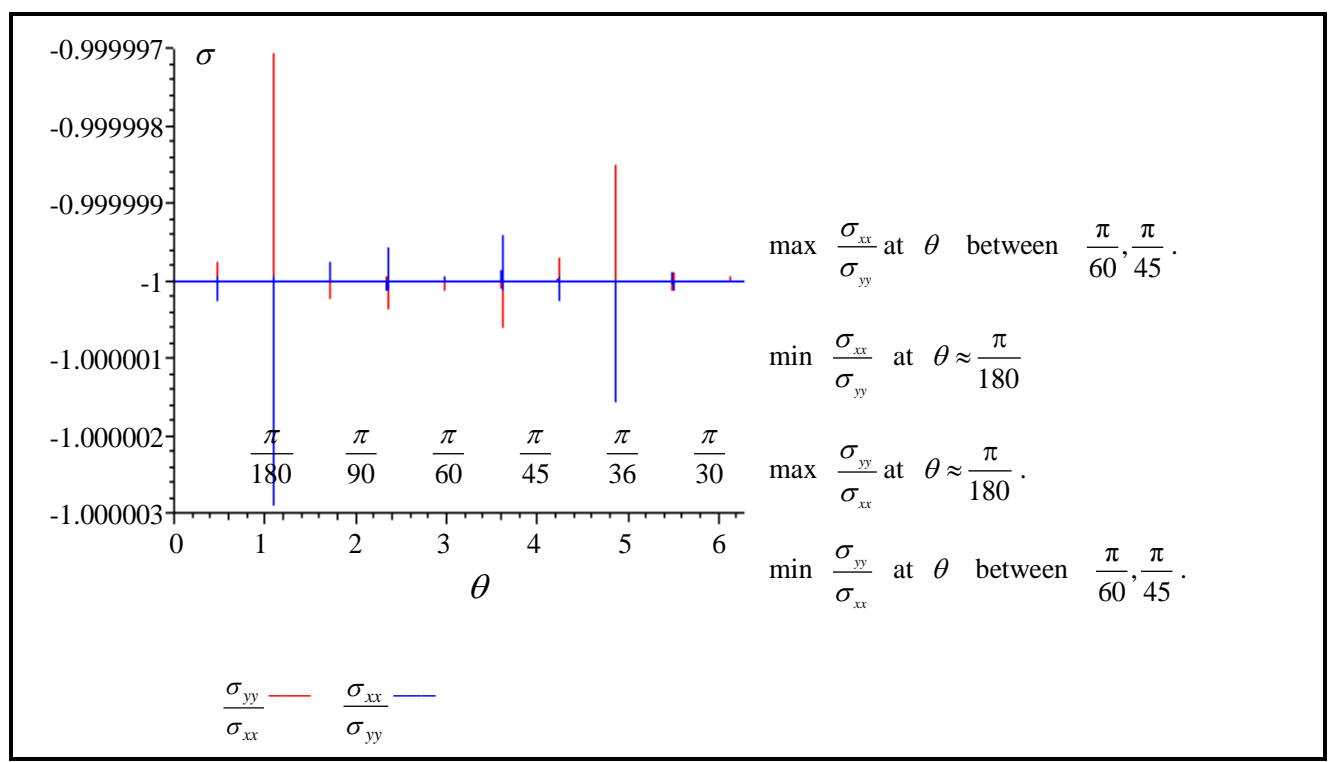

Figure 8. The ratio of vertical to horizontal stresses. 


$$
\begin{aligned}
& c b=\frac{\chi h v n(X+i Y)-n h^{2} v^{2}(X-i Y)}{2 \pi(1+\chi)\left(\chi^{2}-h^{2} v^{2}\right)} \\
& -\chi \phi(\zeta)=\frac{h}{(n-\zeta)}\left(\frac{\chi h v n(X+i Y)-n h^{2} v^{2}(X-i Y)}{2 \pi(1+\chi)\left(\chi^{2}-h^{2} v^{2}\right)}-\frac{n(X-i Y)}{2 \pi(1+\chi)}\right) \\
& \phi(\zeta)=\frac{-1}{\chi} \frac{h n}{2 \pi(1+\chi)(n-\zeta)}\left(\frac{\chi h v(X+i Y)}{\left(\chi^{2}-h^{2} v^{2}\right)}-(X-i Y)\left(1+\frac{h^{2} v^{2}}{\left(\chi^{2}-h^{2} v^{2}\right)}\right)\right) \\
& \psi(\zeta)=-\frac{w\left(\zeta^{-1}\right)}{w^{\prime}(\zeta)} \phi_{*}(\zeta)+\frac{h \zeta}{(1-n \zeta)} \phi_{*}\left(n^{-1}\right)
\end{aligned}
$$

where

$$
\phi_{*}(\zeta)=\phi^{\prime}(\zeta)-\frac{(X+i Y)}{2 \pi(1+\chi) \zeta}
$$

\section{References}

[1] Muskhelishvili, N.I. (1953) Some Basic Problems of Mathematical Theory of Elasticity. Noordroof, Holland.

[2] Spiegel. M.R. (1964) Theory and Problems of Complex Variables, Schaum's Outline Series. McGraw-Hill, New York.

[3] Rubenfeld, L.A. (1985) A First Course in Applied Complex Variables. John Wiley \& Sons, New York.

[4] Bieberbach, L. (1953) Conformal Mapping. Chelsea Publishing Company, New York.

[5] Popov, G.Ya. (1982) Contact Problems for a Linearly Deformable Functions. Odessa, Kiev.

[6] Sabbah, A.S., Abdou, M.A. and Ismail, A.S. (2002) An Infinite Plate with a Curvilinear Hole and Flowing Heat. Proc. Math. Phys. Soc. Egypt.

[7] Atkin, R.J. and Fox, N. (1990) An Introduction to the Theory of Elasticity. Longman, Harlow.

[8] Colton, D. and Kress, R. (1983) Integral Equation Method in Scattering Theory. John Wiley, New York.

[9] Abdou, M.A. (2003) On Asymptotic Method for Fredholm-Volterra Integral Equation of the Second Kind in Contact Problems. Journal of Computational and Applied Mathematics, 154, 431-446. http://dx.doi.org/10.1016/S0377-0427(02)00862-2

[10] Noda, N. and Hetnarski Yoshinobu Tanigowa, R.B. (2003) Thermal Stresses. Taylor and Francis, UK.

[11] Hetnarski, R.B. (2004) Mathematical Theory of Elasticity. Taylor and Francis, London.

[12] Parkus, H. (1976) Thermoelasticity. Springer-Verlag, New York.

[13] Gakhov, F.D. (1966) Boundary Value Problems. General Publishing Company, Ltd., Canada

[14] Ciarlet, P.G., Schultz, M.H. and Varga, R.S. (1967) Numerical Methods of High-Order Accuracy for Nonlinear Boundary Value Problems. I. One Dimensional Problem. Numerische Mathematik, 9, 394-430.

[15] Zebib, A. (1984) A Chebyshev Method for the Solution of Boundary Value Problems. Journal of Computational Physics, 53, 443-455. http://dx.doi.org/10.1016/0021-9991(84)90070-6

[16] Saito, S. and Yamamto, M. (1989) Boundary Value Problems of Quasilinear Ordinary Differential Systems on a Finite Interval. Math. Japon., 34, 447-458.

[17] Abdou, M.A. (1994) First and Second Fundamental Problems for an Elastic Infinite Plate with a Curvilinear Hole. Alex. Eng. J., 33, 227-233.

[18] Abdou, M.A. (2002) Fundamental Problems for an Infinite Plate with a Curvilinear Hole Having Infinite Poles. Applied Mathematics and Computation, 125, 177-193.

[19] Abdou, M.A. and Khamis, A.K. (2000) On a Problem of an Infinite Plate with a Curvilinear Hole Having Three Poles and Arbitrary Shape. Bulletin of the Calcutta Mathematical Society, 92, 309-322.

[20] Abd-Alla, A.M., Abo-Dahab, S.M. and Bayones, F.S. (2013) Propagation of Rayleigh Waves in Magneto-ThermoElastic Half-Space of a Homogeneous Orthotropic Material under the Effect of Rotation, Initial Stress and Gravity 
Field. Journal of Vibration and Control, 19, 1395-1420. http://dx.doi.org/10.1177/1077546312444912

[21] Abd-Alla, A.M. and Abo-Dahab, S.M. (2012) Effect of Rotation and Initial Stress on an Infinite Generalized MagnetoThermoelastic Diffusion Body with a Spherical Cavity. Journal of Thermal Stresses, 35, 892-912. http://dx.doi.org/10.1080/01495739.2012.720209

[22] Abd-Alla, A.M., Abd-Alla, A.N. and Zeidan, N.A. (2000) Thermal Stresses in a Non-Homogeneous Orthotropic Elastic Multilayered Cylinder. Journal of Thermal Stresses, 23, 413-428.

[23] Abd-Alla, A.M., Mahmoud, S.R., Abo-Dahab, S.M. and Helmy, M.I. (2011) Propagation of S-Wave in a Non-Homogeneous Anisotropic Incompressible and Initially Stressed Medium under Influence of Gravity Field. Applied Mathematics and Computation, 217, 4321-4332. http://dx.doi.org/10.1016/j.amc.2010.10.029

[24] Abd-Alla, A.M., Mahmoud, S.R. and AL-Shehri, N.A. (2011) Effect of the Rotation on a Non-Homogeneous Infinite Cylinder of Orthotropic Material. Applied Mathematics and Computation, 217, 8914-8922. 
Scientific Research Publishing (SCIRP) is one of the largest Open Access journal publishers. It is currently publishing more than 200 open access, online, peer-reviewed journals covering a wide range of academic disciplines. SCIRP serves the worldwide academic communities and contributes to the progress and application of science with its publication.

Other selected journals from SCIRP are listed as below. Submit your manuscript to us via either submit@scirp.org or Online Submission Portal.
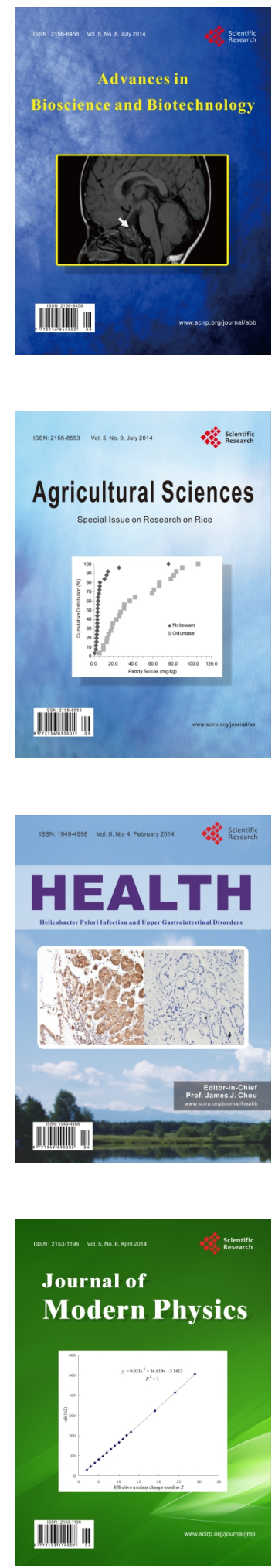
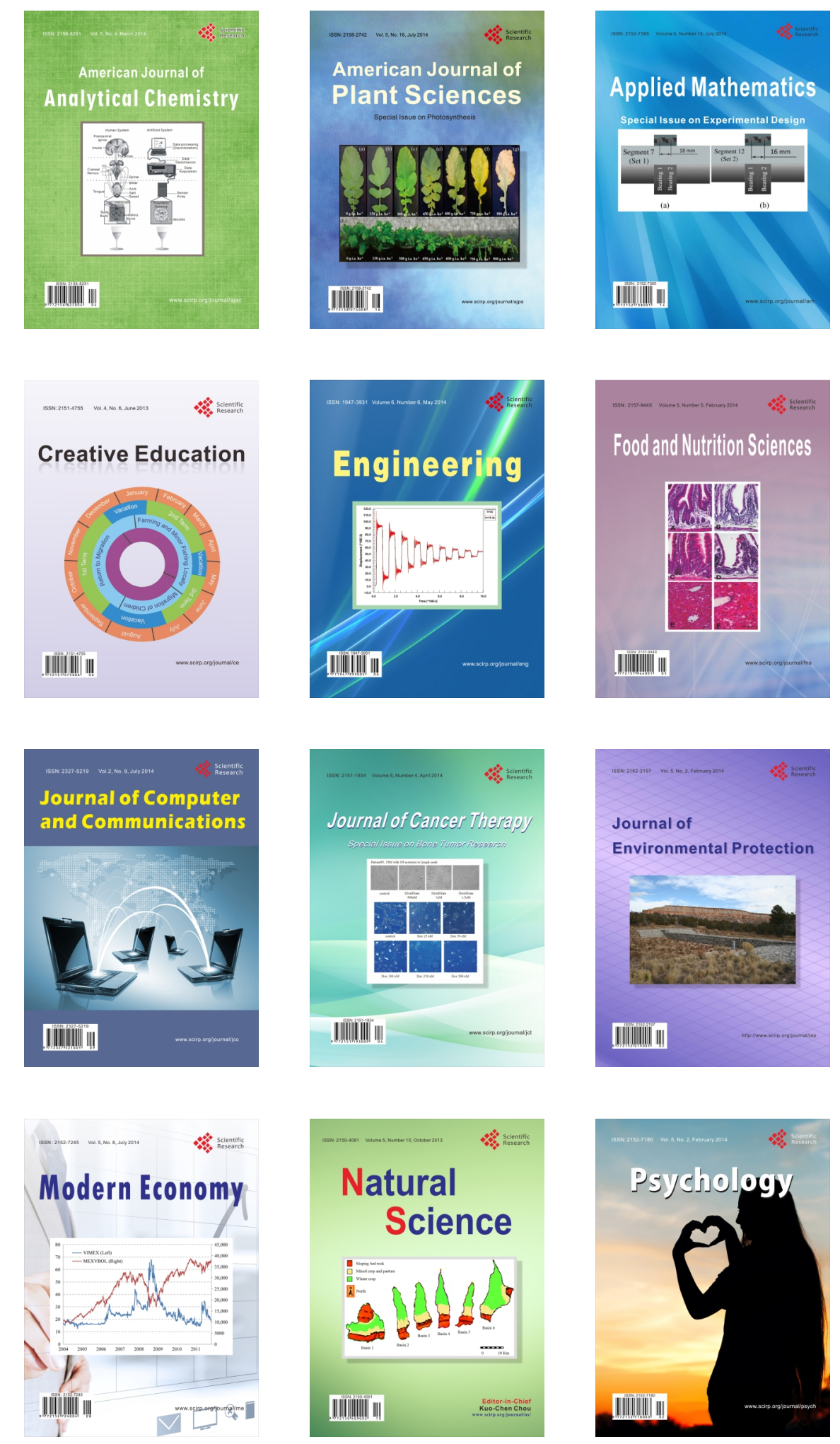